\title{
Efficacy of a Device-Based Continuous Optimization Algorithm for Patients With Cardiac Resynchronization Therapy
}

\author{
Nobuhiko Ueda, MD; Takashi Noda, MD, PhD; Kohei Ishibashi, MD, PhD; Kenzaburo Nakajima, MD; \\ Naoya Kataoka, MD, PhD; Tsukasa Kamakura, MD, PhD; Mitsuru Wada, MD; \\ Kenichiro Yamagata, MD, PhD; Yuko Inoue, MD, PhD; Koji Miyamoto, MD, PhD; \\ Satoshi Nagase, MD, PhD; Takeshi Aiba, MD, PhD; Hideaki Kanzaki, MD, PhD; Chisato Izumi, MD, PhD; \\ Teruo Noguchi, MD, PhD; Satoshi Yasuda, MD, PhD; Kengo Kusano, MD, PhD
}

\begin{abstract}
Background: Cardiac resynchronization therapy (CRT) is less effective in patients with mildly wide QRS or non-left bundle branch block (non-LBBB). A new algorithm of every minute's optimization (adaptive CRT: aCRT algorithm) is effective in patients with CRT devices. This study investigated the clinical effect of the aCRT algorithm, especially in mildly wide QRS $(120 \leq Q R S<150 \mathrm{~ms})$ or nonLBBB patients receiving CRT.
\end{abstract}

\begin{abstract}
Methods and Results: This study included 104 CRT patients (48 patients using the aCRT algorithm [adaptive group] and 56 patients not using the aCRT algorithm [non-adaptive group]). The primary endpoint was a composite clinical outcome of cardiac death and/or heart failure (HF) hospitalization. During a median follow-up of 700 days (interquartile range 362-1,173 days), aCRT reduced the risk of the clinical outcome, even in patients with mildly wide QRS or non-LBBB (log-rank P=0.0030 and $P=0.0077$, respectively) by Kaplan-Meier analysis. Use of the aCRT algorithm was an independent predictor of clinical outcomes in the multivariate analysis (hazard ratio $(\mathrm{HR}) 0.28,95 \%$ confidence interval $(\mathrm{Cl}): 0.096-0.78, \mathrm{P}=0.015)$, the same as in patients with mildly wide QRS (HR 0.12, 95\% Cl: 0.006-0.69, P=0.015).
\end{abstract}

Conclusions: The new aCRT algorithm was useful and significantly reduced the risk of the clinical outcome, even in patients with mildly wide QRS.

Key Words: Adaptive cardiac resynchronization therapy; Algorithms; Heart failure; Mildly wide QRS; Non-left bundle branch block

C ardiac resynchronization therapy (CRT) provides substantial clinical benefit in patients with severe left ventricular (LV) dysfunction and QRS prolongation. Various studies of CRT have demonstrated reductions in mortality and hospitalization; however, up to one-third of patients fail to respond to CRT. ${ }^{1-3}$ Ischemic cardiomyopathy (ICM), large scar burden, and male sex are reported to be factors in the non-response to CRT, and suboptimal atrioventricular (AV) timing is also reported as a potential reason for suboptimal response. ${ }^{4}$ In contrast, left bundle branch block (LBBB) morphology and wide QRS are revealed as predictors of response to CRT.

A new algorithm of every minute's optimization (adaptive CRT: aCRT) provides ambulatory adjustment of pacing configuration (synchronized LV (sLV) pacing or biventricular (BiV) pacing) and continuous pacing timings

\section{Editorial p 11}

of $\mathrm{AV}$ and interventricular (VV) delays based on periodic automatic evaluation of electrical conduction. ${ }^{5}$ Right ventricular (RV) sLV pacing is selected when AV conduction is normal; in contrast, $\mathrm{BiV}$ pacing is selected when $\mathrm{AV}$ conduction is prolonged. A previous study demonstrated that the aCRT algorithm is associated with a significant reduction in 30-day readmission, both after heart failure (HF) and all-cause hospitalization. ${ }^{6}$ Little is known regarding the clinical impact, including on mortality and HF hospitalization, of the aCRT algorithm, however, especially in mildly wide QRS $(120 \leq \mathrm{QRS}<150 \mathrm{~ms})$ or nonLBBB patients. The purpose of this study was to examine the clinical impact of the aCRT algorithm in patients with

Received July 31, 2019; revised manuscript received September 20, 2019; accepted September 24, 2019; J-STAGE Advance Publication released online October 26, 2019 Time for primary review: 14 days

Department of Cardiovascular Medicine, National Cerebral and Cardiovascular Center, Suita (N.U., T. Noda, K.I., K.N., N.K., T.K., M.W., K.Y., Y.I., K.M., S.N., T.A., H.K., C.I., T. Noguchi, S.Y., K.K.); Department of Advanced Cardiovascular Medicine, Graduate School of Medical Sciences, Kumamoto University, Kumamoto (N.U., K.N., M.W., S.Y.), Japan

Mailing address: Takashi Noda, MD, PhD, FJCS, Department of Cardiovascular Medicine, National Cerebral and Cardiovascular Center, 6-1 Kishibe-Shimmachi, Suita 564-8565, Japan. E-mail: tnoda@ncvc.go.jp

ISSN-1346-9843 All rights are reserved to the Japanese Circulation Society. For permissions, please e-mail: cj@j-circ.or.jp 


\begin{tabular}{|c|c|c|c|c|}
\hline Clinical characteristics & $\begin{array}{c}\text { All } \\
(n=104)\end{array}$ & $\begin{array}{c}\text { Adaptive } \\
(n=48)\end{array}$ & $\begin{array}{c}\text { Non-adaptive } \\
(n=56)\end{array}$ & $P$ value \\
\hline Age (years) & $62.1 \pm 13.9$ & $64.1 \pm 12.3$ & $60.4 \pm 15.0$ & 0.17 \\
\hline Male, n (\%) & $74(71)$ & $34(71)$ & $40(71)$ & 0.95 \\
\hline CRT-D, n (\%)/CRT-P, n (\%) & $95(91) / 9(16)$ & $48(100) / 0(0)$ & $47(84) / 9(16)$ & 0.0006 \\
\hline Secondary prevention, n (\%) & $23(22)$ & $10(21)$ & $13(23)$ & 0.77 \\
\hline \multicolumn{5}{|l|}{ Medical history } \\
\hline ICM, n (\%)/NICM, n (\%) & $18(17) / 86(83)$ & $11(23) / 37(77)$ & $7(13) / 49(88)$ & 0.16 \\
\hline $\mathrm{PAF}, \mathrm{n}(\%)$ & $31(30)$ & $14(29)$ & $17(30)$ & 0.89 \\
\hline \multicolumn{5}{|l|}{ Medications } \\
\hline$\beta$-blockers, n (\%) & $96(92)$ & $46(96)$ & $50(89)$ & 0.20 \\
\hline Amiodarone, n (\%) & $45(43)$ & $22(46)$ & $23(41)$ & 0.63 \\
\hline \multicolumn{5}{|l|}{ Echocardiography } \\
\hline LVDd (mm) & $65.8 \pm 10.8$ & $65.4 \pm 10.6$ & $66.1 \pm 11.1$ & 0.72 \\
\hline LVDs (mm) & $57.3 \pm 12.5$ & $56.6 \pm 11.6$ & $57.8 \pm 13.2$ & 0.65 \\
\hline LVEF (\%) & $24.6 \pm 9.4$ & $25.4 \pm 8.3$ & $23.9 \pm 10.3$ & 0.43 \\
\hline$E / A$ & $1.8 \pm 1.4$ & $1.8 \pm 1.4$ & $1.9 \pm 1.4$ & 0.69 \\
\hline DcT (ms) & $176 \pm 58$ & $177 \pm 65$ & $173 \pm 41$ & 0.84 \\
\hline \multicolumn{5}{|l|}{ Electrocardiography } \\
\hline LBBB, n (\%)/non-LBBB, n (\%) & $29(28) / 75(72)$ & $15(31) / 33(69)$ & $14(25) / 42(75)$ & 0.48 \\
\hline QRS duration (ms) & $159.2 \pm 33.9$ & $154.6 \pm 32.3$ & $163.1 \pm 35.1$ & 0.20 \\
\hline$P Q$ interval (ms) & $174.8 \pm 90.7$ & $161.0 \pm 80.3$ & $185.9 \pm 98.0$ & 0.27 \\
\hline Responder, n (\%) & $65(63)$ & $32(67)$ & $33(59)$ & 0.42 \\
\hline
\end{tabular}

CRT-D, cardiac resynchronization therapy defibrillator; CRT-P, cardiac resynchronization therapy pacemaker; DcT, deceleration time; E/A, ratio of mitral peak flow velocity in early diastole to peak flow velocity in late diastole; ICM, ischemic cardiomyopathy; LBBB, left bundle branch block; LVDd, left ventricular diastolic dimension; LVDs, left ventricular systolic dimension; LVEF, left ventricular ejection fraction; NICM, nonischemic cardiomyopathy; PAF, paroxysmal atrial fibrillation.

mildly wide QRS (120-150ms) or non-LBBB morphology.

\section{Methods}

\section{Study Population}

This study was a retrospective single-center cohort study. We investigated 104 patients with a CRT device (Medtronic Inc., Minneapolis, MN, USA) who underwent implantation between 2014 and 2018. Medtronic devices were implanted in all patients. The details of device type were: 71 patients (adaptive: 30 patients, non-adaptive 41 patients) had a Viva ${ }^{\circledR}$ series; 3 patients (all non-adaptive) had a Protecta ${ }^{\circledR}$ series; 3 patients (all non-adaptive) had a Consulta CRT-P ${ }^{\circledR} ; 11$ patients (adaptive: 9 patients, nonadaptive: 2 patients) had a Claria MRI Quad ${ }^{\circledR}$; and 16 patients (adaptive: 9 patients, non-adaptive: 7 patients) had an Amplia MRI Quad ${ }^{\circledR}$. Inclusion criteria for CRT were based on the Japanese guideline, and all patients had QRS width $\geq 120 \mathrm{~ms}$ and LV ejection fraction (LVEF) $\leq 35 \%$. The adaptive group consisted of 48 consecutive CRT patients using the aCRT algorithm. The non-adaptive group consisted of 56 consecutive CRT patients not using the aCRT algorithm during the same time period. Whether or not the aCRT algorithm was used depended on the physician who implanted the device. Patients with permanent atrial fibrillation (AF), a LV assist device, acute cardiac failure, recent myocardial infarction, coronary artery bypass graft surgery within 3 months, or severe aortic stenosis were excluded. This study was approved by the Institutional Research Board of the National Cerebral and Cardiovascular Center, Suita, Japan (M26-150-7).

\section{Device Implantation and Programming}

CRT devices were implanted transvenously in the standard fashion after obtaining written informed consent from all patients or their guardians. An RV lead was positioned at the RV septal apex, and then the LV lead was implanted in a suitable lateral, posterolateral, or anterolateral branch of the coronary vein at a site that produced an acceptable pacing threshold without diaphragmatic pacing. The aCRT algorithm was programmed from the day of implantation.

The details of the aCRT algorithm have been published previously. ${ }^{5}$ In brief, if the conduction interval from the right atrium to the $\mathrm{RV}$ is normal (intrinsic $\mathrm{AV}$ interval $\leq 220 \mathrm{~ms}$, if in sinus rhythm or AV interval $\leq 270 \mathrm{~ms}$, if receiving atrial pacing) and the heart rate does not exceed 100 beats/min, the algorithm provides sLV. Conversely, if the intrinsic AV conduction interval is prolonged, the algorithm provides $\mathrm{BiV}$. The timing of $\mathrm{LV}$ pacing is programmed as $70 \%$ of the intrinsic atrial pacing to the RV sensing (RVs) interval ${ }^{7}$ or $40 \mathrm{~ms}$ shorter than the intrinsic PQ interval. ${ }^{8}$ The algorithm switches to BiV pacing with the AV delay calculated from the intrinsic intervals between atrial sensing and RVs (A-RVs) and between atrial sensing and the end of the $P$ wave (A-Pend) to pace after the completion of the $\mathrm{P}$ wave but prior to intrinsic RVs by at least $50 \mathrm{~ms} .{ }^{9}$ In the non-adaptive group, echocardiographic optimization was performed with initial AV delay optimization based on patient transmitral flow patterns. Next, VV delay adjustment to produce the greatest aortic velocity time integral was carried out by the iterative method. ${ }^{10,11}$ Two patients in the adaptive group, but none 
Table 2. Comparison of the Characteristics of Patients in the Adaptive CRT ON Group (Adaptive) and the Adaptive CRT OFF Group (Non-Adaptive) With a Mildly Wide QRS

\begin{tabular}{|c|c|c|c|c|}
\hline Clinical characteristics & $\begin{array}{c}\text { All } \\
(n=42)\end{array}$ & $\begin{array}{c}\text { Adaptive } \\
(n=24)\end{array}$ & $\begin{array}{l}\text { Non-adaptive } \\
\quad(n=18)\end{array}$ & $P$ value \\
\hline Age (years) & $57.9 \pm 15.6$ & $62.3 \pm 13.8$ & $52.1 \pm 16.3$ & 0.033 \\
\hline Male, n (\%) & $32(76)$ & $19(79)$ & $13(72)$ & 0.60 \\
\hline CRT-D, n (\%)/CRT-P, n (\%) & $41(98) / 1(2)$ & $24(100) / 0(0)$ & $17(94) / 1(6)$ & 0.19 \\
\hline Secondary prevention, n (\%) & $5(12)$ & $4(17)$ & $1(6)$ & 0.25 \\
\hline \multicolumn{5}{|l|}{ Medical history } \\
\hline ICM, n (\%)/NICM, n (\%) & $8(19) / 34(81)$ & $5(21) / 19(79)$ & $3(17) / 15(83)$ & 0.73 \\
\hline PAF, n (\%) & $15(36)$ & $9(38)$ & $6(33)$ & 0.78 \\
\hline \multicolumn{5}{|l|}{ Medications } \\
\hline$\beta$-blockers, $\mathrm{n}(\%)$ & $41(95)$ & $24(100)$ & $17(94)$ & 0.19 \\
\hline Amiodarone, n (\%) & $17(40)$ & $11(46)$ & $6(33)$ & 0.41 \\
\hline \multicolumn{5}{|l|}{ Echocardiography } \\
\hline LVDd (mm) & $65.0 \pm 9.3$ & $64.3 \pm 9.1$ & $66.0 \pm 9.6$ & 0.57 \\
\hline LVDs (mm) & $56.2 \pm 11.5$ & $54.9 \pm 10.6$ & $57.9 \pm 12.7$ & 0.41 \\
\hline LVEF (\%) & $25.0 \pm 10.5$ & $25.5 \pm 9.7$ & $24.5 \pm 11.8$ & 0.77 \\
\hline$E / A$ & $1.9 \pm 1.4$ & $1.9 \pm 1.4$ & $2.0 \pm 1.5$ & 0.81 \\
\hline $\mathrm{DcT}(\mathrm{ms})$ & $180 \pm 57$ & $180 \pm 62$ & $180 \pm 49$ & 0.98 \\
\hline \multicolumn{5}{|l|}{ Electrocardiography } \\
\hline LBBB, n (\%)/non-LBBB, n (\%) & $12(29) / 30(71)$ & $5(21) / 19(79)$ & $7(39) / 11(61)$ & 0.20 \\
\hline QRS duration (ms) & $126.6 \pm 17.1$ & $128.9 \pm 14.9$ & $123.4 \pm 19.7$ & 0.31 \\
\hline$P Q$ interval (ms) & $144.8 \pm 100.7$ & $148.0 \pm 97.0$ & $140.8 \pm 109.3$ & 0.86 \\
\hline Responder, n (\%) & $21(50)$ & $13(54)$ & $8(44)$ & 0.53 \\
\hline
\end{tabular}

Abbreviations as in Table 1.

Table 3. Comparison of the Characteristics of Patients in the Adaptive CRT ON Group (Adaptive) and the Adaptive CRT OFF Group (Non-Adaptive) Without LBBB or Right Ventricular Pacing Morphology

\begin{tabular}{|c|c|c|c|c|}
\hline Clinical characteristics & $\begin{array}{c}\text { All } \\
(n=43)\end{array}$ & $\begin{array}{l}\text { Adaptive } \\
(\mathrm{n}=17)\end{array}$ & $\begin{array}{l}\text { Non-adaptive } \\
\quad(n=26)\end{array}$ & $P$ value \\
\hline Age (years) & $58.2 \pm 14.7$ & $58.4 \pm 14.8$ & $58.1 \pm 14.9$ & 0.95 \\
\hline Male, $\mathrm{n}(\%)$ & $29(67)$ & $12(71)$ & $17(65)$ & 0.72 \\
\hline CRT-D, n (\%)/CRT-P, n (\%) & $41(95) / 2(5)$ & $17(100) / 0(0)$ & $24(92) / 2(8)$ & 0.15 \\
\hline Secondary prevention, $\mathrm{n}(\%)$ & $11(26)$ & $5(29)$ & $6(23)$ & 0.64 \\
\hline \multicolumn{5}{|l|}{ Medical history } \\
\hline ICM, n (\%)/NICM, n (\%) & $8(19) / 35(81)$ & $4(24) / 13(76)$ & $4(15) / 22(85)$ & 0.51 \\
\hline PAF, n (\%) & $16(37)$ & $6(35)$ & $10(38)$ & 0.83 \\
\hline \multicolumn{5}{|l|}{ Medications } \\
\hline$\beta$-blockers, n (\%) & $39(91)$ & $16(94)$ & $23(88)$ & 0.52 \\
\hline Amiodarone, n (\%) & $20(47)$ & $8(47)$ & $12(46)$ & 0.95 \\
\hline \multicolumn{5}{|l|}{ Echocardiography } \\
\hline $\operatorname{LVDd}(\mathrm{mm})$ & $63.7 \pm 9.3$ & $62.8 \pm 8.4$ & $64.2 \pm 10.0$ & 0.64 \\
\hline LVDs (mm) & $54.4 \pm 11.8$ & $53.1 \pm 10.6$ & $55.3 \pm 12.7$ & 0.56 \\
\hline LVEF (\%) & $26.7 \pm 11.4$ & $25.9 \pm 10.5$ & $27.3 \pm 12.2$ & 0.69 \\
\hline $\mathrm{E} / \mathrm{A}$ & $2.5 \pm 1.7$ & $2.6 \pm 2.0$ & $2.5 \pm 1.4$ & 0.93 \\
\hline $\mathrm{DcT}(\mathrm{ms})$ & $179 \pm 54$ & $175 \pm 58$ & $185 \pm 50$ & 0.67 \\
\hline \multicolumn{5}{|l|}{ Electrocardiography } \\
\hline IVCD, n (\%)/RBBB, n (\%) & $28(65) / 15(35)$ & $15(88) / 2(12)$ & $13(50) / 13(50)$ & 0.0071 \\
\hline QRS duration (ms) & $144.4 \pm 26.8$ & $134.4 \pm 16.3$ & $151.0 \pm 30.3$ & 0.045 \\
\hline$P Q$ interval (ms) & $155.1 \pm 98.9$ & $141.0 \pm 100.3$ & $163.0 \pm 100.1$ & 0.58 \\
\hline Responder, n (\%) & $21(49)$ & $9(53)$ & $12(46)$ & 0.66 \\
\hline
\end{tabular}

IVCD, intraventricular conduction disturbance; RBBB, right bundle branch block. Other abbreviations as in Table 1. 

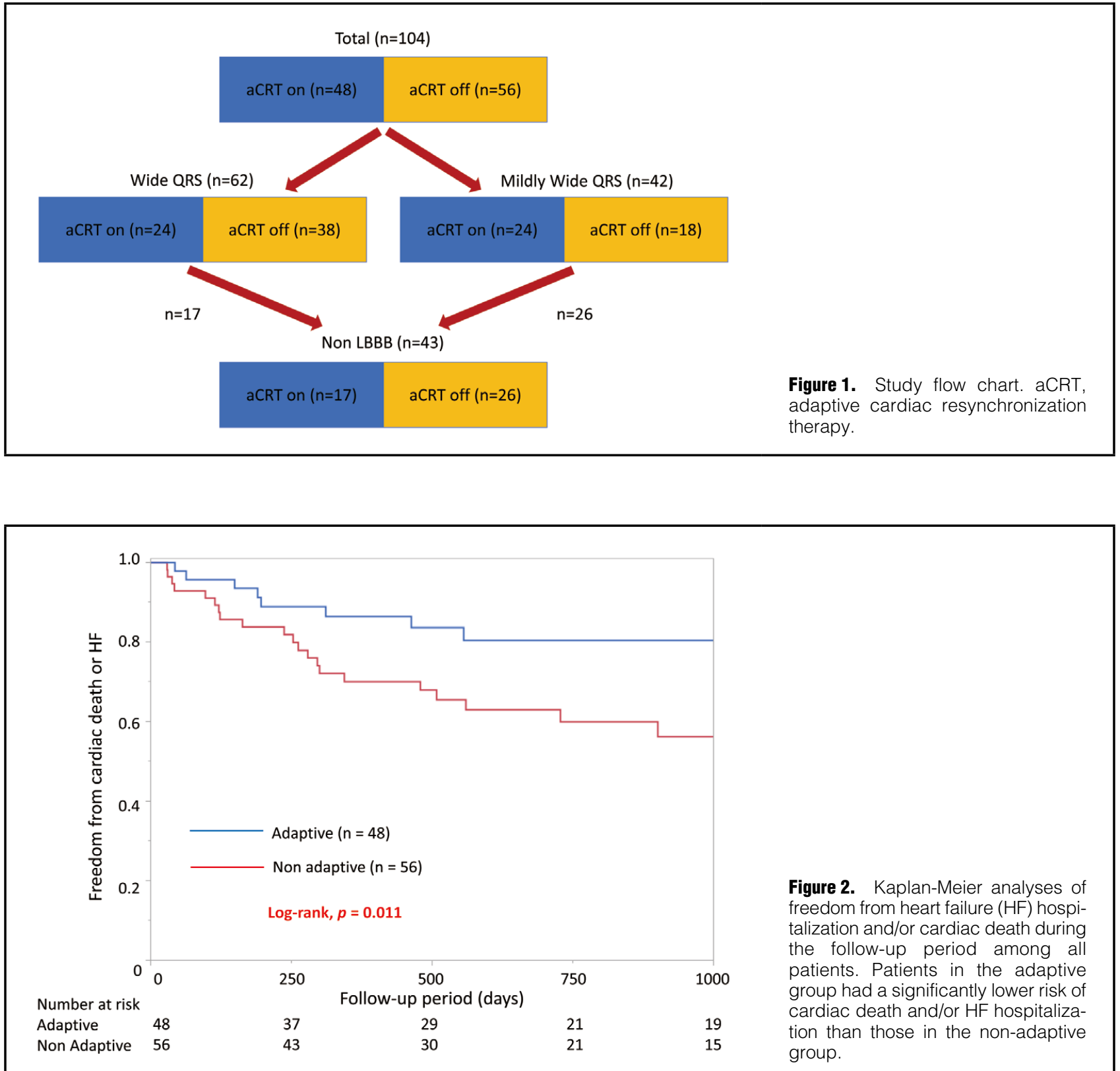

Figure 2. Kaplan-Meier analyses of freedom from heart failure (HF) hospitalization and/or cardiac death during the follow-up period among all patients. Patients in the adaptive group had a significantly lower risk of cardiac death and/or HF hospitalization than those in the non-adaptive group.

of the patients in non-adaptive group, used multipoint pacing. There were no differences in the parameters of ventricular arrhythmia (VA) detection. Among the patients for secondary prevention, we programmed ventricular tachycardia (VT), fast VT, and the ventricular fibrillation detection zone according to the cycle length of the VA. We used a long detection interval (e.g., 30/40 number of internal detections) and a high detection rate (e.g., fast VT $\geq 182$ beats/min), especially in patients for primary prevention.

\section{Clinical Data}

Clinical data were collected for all patients, including age, sex, underlying heart disease, history of AF, medications, echocardiographic data such as LVEF, ratio of mitral peak velocity in early diastole to peak flow velocity in late diastole (E/A), deceleration time (DcT), and ECG data such as QRS morphology, QRS duration, and PQ interval. LBBB was defined as intrinsic QRS duration $\geq 120 \mathrm{~ms}$ with a broad notched or slurred $R$ wave in leads I, V5, and V6, and occasional RS pattern in V5 and V6. In addition, Q waves were absent in leads I, V5, and V6, and R peak time was $>60 \mathrm{~ms}$ in leads V5 and V6 but normal in leads V1-3. ${ }^{12}$ The widest QRS duration on each 12-lead ECG was manually measured by an expert cardiologist, who was blinded to the patient's data. Mildly wide QRS was defined as a QRS duration between 120 and $150 \mathrm{~ms}$. Echocardiographic data were collected before and at least 6 months after CRT implantation. A CRT responder was defined as a patient with an improvement in LVEF $\geq 10 \%$ and/or a reduction in LV end-systolic volume (LVESV) $\geq 15 \%$ compared with baseline at 6 months after CRT device implantation, which was the same as the definition of a positive CRT response. ${ }^{\mathbf{1 3}, 14} \mathrm{We}$ also evaluated the incidence of cardiac death and HF hospitalization. 

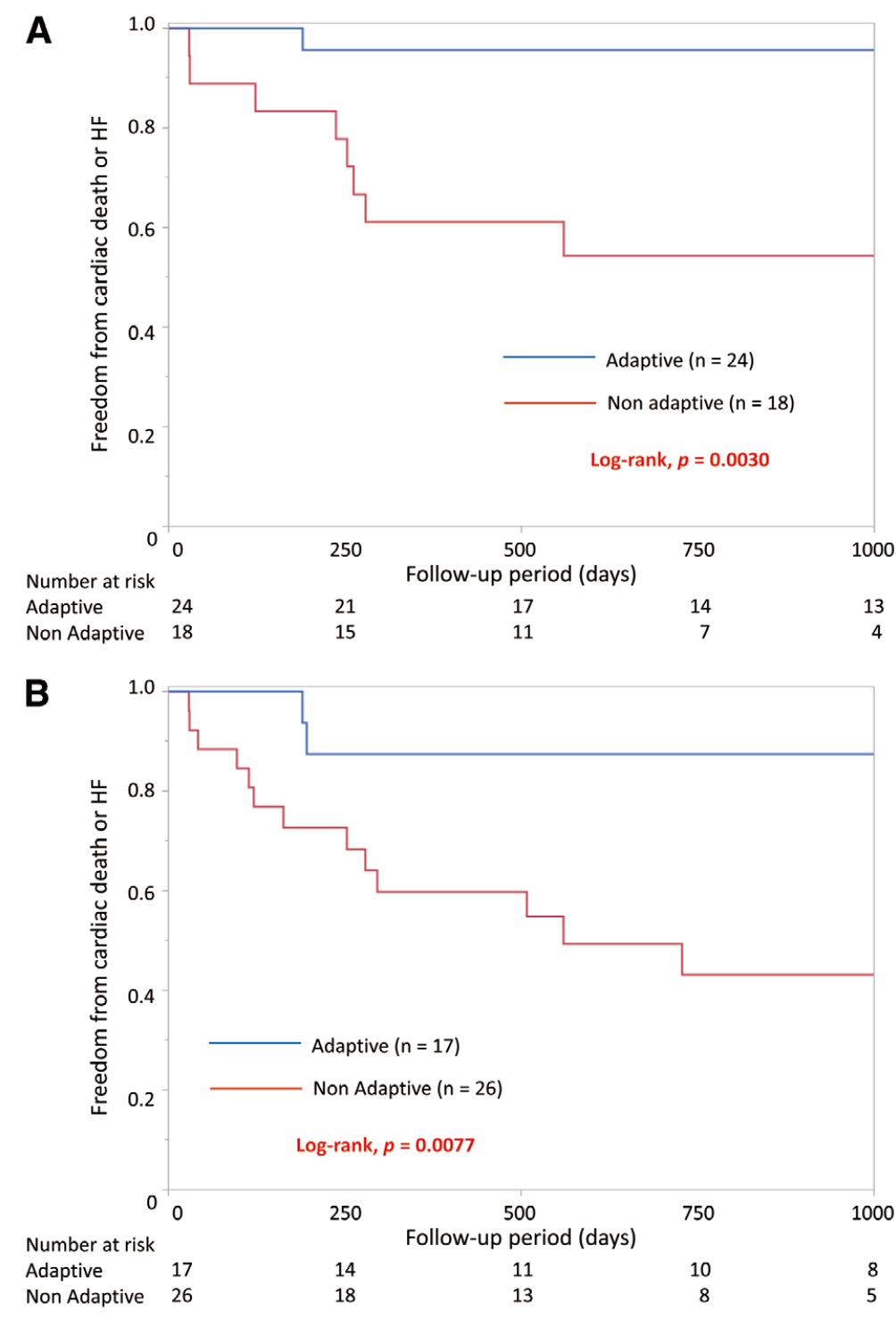

Figure 3. Kaplan-Meier analyses of freedom from heart failure (HF) hospitalization and/or cardiac death during the follow-up period in patients with a mildly wide QRS $(\mathbf{A})$ or nonLBBB morphology (B). Patients in the adaptive group had a lower risk of cardiac death and/or HF than patients in the non-adaptive group. LBBB, left bundle branch block.

\section{Statistical Analysis}

The numeric values are presented as mean \pm standard deviation. The categorical data are expressed as counts and percentages. Categorical differences between groups were evaluated by chi-squared test or Fisher's exact test as appropriate. Continuous variables were compared using the Wilcoxon rank-sum test or the Kruskal-Wallis test. A value of $\mathrm{P}<0.05$ was taken as a threshold for statistical significance. All analyses were performed using JMP 12 software (SAS Institute Inc., Cray, NC, USA).

\section{Results}

Clinical Characteristics

Patients' clinical characteristics are shown in Tables 1-3. Among 104 patients, 74 (71\%) were male. Patients were $62 \pm 14$ years old. In terms of the etiology of underlying heart disease, $18(17 \%)$ patients with ICM and $86(83 \%)$ with non-ICM were included. With respect to medications, 96 patients $(92 \%)$ were prescribed beta-blockers and 45
(43\%) patients were prescribed amiodarone. Mean LVEF was $25 \pm 9 \%$. As for comparisons between the adaptive and non-adaptive groups, there were no significant differences in age, sex, indication for defibrillator, and medications. Regarding the ECG parameters, the mean PQ interval was $<200 \mathrm{~ms}$ in both groups. A total of $24(50 \%)$ patients in the adaptive group and $18(32 \%)$ in the non-adaptive group had a mildly wide QRS and $17(35 \%)$ patients in the adaptive group and $26(46 \%)$ patients in the non-adaptive group had non-LBBB and without RV pacing (Figure 1). In addition, there was no significant difference in the prevalence of responders $(\mathrm{P}=0.42)$ (Table 1). In the 42 patients with a mildly wide $\mathrm{QRS}$, there were no significant differences in clinical characteristics except for age (Table 2). Among patients with non-LBBB morphology only, the proportion of intraventricular conduction delay (IVCD) as QRS morphology was significantly higher in the adaptive group than in the non-adaptive group. In addition, the QRS duration was significantly narrower in the adaptive group compared with the non-adaptive group (Table 3). 


\begin{tabular}{|c|c|c|c|c|c|c|}
\hline & \multicolumn{3}{|c|}{ Univariate analysis } & \multicolumn{3}{|c|}{ Multivariate analysis } \\
\hline & HR & $95 \% \mathrm{Cl}$ & $P$ value & HR & $95 \% \mathrm{Cl}$ & $P$ value \\
\hline Age & 0.99 & $0.96-1.01$ & 0.31 & & & \\
\hline Sex (male) & 0.70 & $0.34-1.51$ & 0.34 & & & \\
\hline CRT-D & 2.80 & $0.60-49.9$ & 0.23 & & & \\
\hline Secondary prevention & 1.18 & $0.49-2.53$ & 0.69 & & & \\
\hline $\mathrm{ICM}$ & 1.09 & $0.40-2.49$ & 0.85 & & & \\
\hline PAF & 1.68 & $0.79-3.42$ & 0.17 & & & \\
\hline Amiodarone & 1.25 & $0.61-2.55$ & 0.53 & & & \\
\hline LVEF (1\% increase) & 0.96 & $0.92-1.00$ & 0.056 & & & \\
\hline $\mathrm{E} / \mathrm{A}$ & 1.30 & $0.93-1.78$ & 0.12 & & & \\
\hline DcT (1-ms increase) & 0.99 & $0.98-1.00$ & 0.015 & 0.98 & $0.97-1.00$ & 0.0093 \\
\hline LBBB & 0.59 & $0.23-1.32$ & 0.21 & & & \\
\hline QRS duration (1-ms increase) & 1.00 & $0.99-1.01$ & 0.67 & & & \\
\hline$P Q$ interval (1-ms increase) & 1.00 & $1.00-1.01$ & 0.22 & & & \\
\hline Adaptive CRT & 0.37 & $0.15-0.79$ & 0.0096 & 0.28 & $0.096-0.78$ & 0.015 \\
\hline
\end{tabular}

$\mathrm{Cl}$, confidence interval; $\mathrm{HR}$, hazard ratio. Other abbreviations as in Table 1.

\section{Clinical Outcome}

During a median 700 days (interquartile range $362-1,173$ days) of follow-up, 20 patients (adaptive group: 4 patients, non-adaptive group: 16 patients) died of cardiac death, 28 (adaptive group: 8 patients, non-adaptive group: 20 patients) experienced HF hospitalization, and 4 patients (adaptive group: 2 patients, non-adaptive group: 2 patients) had an appropriate shock therapy for VA. The patients in the adaptive group $(n=48)$ had a significantly lower incidence of either cardiac death or HF hospitalization (log-rank, $\mathrm{P}=0.011)$ than those in the non-adaptive group $(\mathrm{n}=56)$ (Figure 2). Even in patients with mildly wide QRS duration only, the adaptive group $(n=24)$ showed a significantly lower incidence of cardiac death or HF hospitalization than the non-adaptive group $(n=18)(\log -r a n k$, $\mathrm{P}=0.0030$, Figure 3A). Among the patients with non-LBBB morphology only, the adaptive group $(n=17)$ also showed a significantly lower incidence of cardiac death or HF hospitalization compared with the non-adaptive group $(n=26)$ $(\mathrm{P}=0.0077$, Figure 3B $)$.

Univariate and multivariate analyses revealed that use of the aCRT algorithm was an independent predictor of cardiac death or HF hospitalization (hazard ratio (HR) $0.28,95 \%$ confidence interval $(\mathrm{CI}): 0.096-0.78, \mathrm{P}=0.015$ ) (Table 4), the same as in patients with a mildly wide QRS (HR 0.12, 95\% CI: 0.006-0.69, P=0.015) (Table 5).

\section{Discussion}

\section{Main Findings}

To the best of our knowledge, this is the first study to evaluate the efficacy of the aCRT algorithm for CRT patients with a mildly wide QRS or non-LBBB morphology. This study demonstrated that the aCRT algorithm was associated with a reduction in cardiac deaths and HF hospitalizations of CRT patients, even those with a mildly wide QRS.

\section{Efficacy of the aCRT Algorithm}

The aCRT algorithm has been reported to be beneficial and safe in some studies. The aCRT trial showed that the
aCRT algorithm resulted in a $44 \%$ absolute reduction in the percentage of RV pacing, and the optimization provided by the aCRT algorithm was non-inferior to echocardiographic optimization with respect to clinical, structural, and functional improvements, such as LVESV index, LVEF, New York Heart Association, 6-min walk distance, and Minnesota Living With Heart Failure quality of life. ${ }^{15}$ In fact, the proportion of improved clinical composite score of the aCRT group was non-inferior to the echocardiographic optimization group. Starling et $\mathrm{al}^{\mathbf{6}}$ evaluated patients who had $\geq 1$ index hospitalizations during the follow-up in the aCRT trial. They showed that use of the aCRT algorithm was associated with a significant reduction in the probability of a 30-day readmission, both after $\mathrm{HF}$ and all-cause hospitalization. In the aCRT trial, however, there were no significant differences with respect to HF events or VA episodes during the follow-up period. Few studies showed a reduction in HF hospitalization or all-cause mortality. Our cohort data revealed that use of the aCRT algorithm was an independent predictor of cardiac death or HF hospitalization, not only in all study subjects $(n=104)$ but also in patients with a mildly wide QRS only.

\section{Possible Mechanism of Effectiveness of the aCRT Algorithm}

The aCRT algorithm can provide RV-sLV pacing and adjust the AV and VV delays based on periodic automatic evaluation of electrical conduction. The reduction in RV pacing by the aCRT algorithm may avoid the deleterious effect of iatrogenic ventricular dyssynchrony. ${ }^{16}$ Appropriately timed LV pacing was reported to be superior to simultaneous BiV pacing in a CRT subpopulation with sinus rhythm and intact $\mathrm{AV}$ conduction. ${ }^{17}$ Thus, in patients with normal AV conduction, the aCRT algorithm could be effective.

In addition, optimal AV delay is reported to change dynamically. O'Donnell et al reported a trend towards an increase in the optimal AV delay for CRT during followup. ${ }^{18}$ Moreover, during exercise, AV delay reduces dramatically and, in this situation, rate-adaptive AV delay 


\begin{tabular}{|c|c|c|c|c|c|c|c|c|c|c|c|c|}
\hline & \multicolumn{6}{|c|}{ Mildly wide QRS } & \multicolumn{6}{|c|}{ Non-LBBB or RV pacing } \\
\hline & \multicolumn{3}{|c|}{ Univariate analysis } & \multicolumn{3}{|c|}{ Multivariate analysis } & \multicolumn{3}{|c|}{ Univariate analysis } & \multicolumn{3}{|c|}{ Multivariate analysis } \\
\hline & HR & $95 \% \mathrm{Cl}$ & $\begin{array}{c}P \\
\text { value }\end{array}$ & HR & $95 \% \mathrm{Cl}$ & $\begin{array}{c}P \\
\text { value }\end{array}$ & HR & $95 \% \mathrm{Cl}$ & $\begin{array}{c}P \\
\text { value }\end{array}$ & HR & $95 \% \mathrm{Cl}$ & $\begin{array}{c}P \\
\text { value }\end{array}$ \\
\hline Age & 0.95 & $0.91-1.00$ & 0.031 & 0.97 & $0.92-1.02$ & 0.25 & 1.00 & $0.97-1.04$ & 0.97 & & & \\
\hline Sex (male) & 0.71 & $0.19-3.39$ & 0.64 & & & & 0.93 & $0.34-2.76$ & 0.89 & & & \\
\hline CRT-D & $6.8^{*} 10^{7}$ & $0.13-0.13$ & 0.47 & & & & 0.47 & $0.091-8.63$ & 0.52 & & & \\
\hline Secondary prevention & 1.06 & $0.057-5.80$ & 0.96 & & & & 2.07 & $0.70-5.61$ & 0.18 & & & \\
\hline ICM & 0.49 & $0.026-2.67$ & 0.46 & & & & 0.92 & $0.21-2.88$ & 0.90 & & & \\
\hline PAF & 1.63 & $0.40-6.19$ & 0.47 & & & & 1.14 & $0.39-3.06$ & 0.81 & & & \\
\hline Amiodarone & 0.79 & $0.17-3.02$ & 0.74 & & & & 1.23 & $0.45-3.36$ & 0.68 & & & \\
\hline LVEF (1\% increase) & 0.93 & $0.85-1.00$ & 0.056 & & & & 0.95 & $0.90-1.00$ & 0.032 & 0.97 & $0.91-1.02$ & 0.24 \\
\hline $\mathrm{E} / \mathrm{A}$ & 1.11 & $0.61-1.85$ & 0.70 & & & & 1.26 & $0.82-1.94$ & 0.28 & & & \\
\hline DcT (1-ms increase) & 0.99 & $0.97-1.01$ & 0.22 & & & & 0.99 & $0.97-1.00$ & 0.11 & & & \\
\hline LBBB & 1.17 & $0.25-4.42$ & 0.83 & & & & & & & & & \\
\hline $\begin{array}{l}\text { QRS duration } \\
\text { (1-ms increase) }\end{array}$ & 1.00 & $0.96-1.04$ & 0.93 & & & & 1.01 & $0.99-1.03$ & 0.29 & & & \\
\hline $\begin{array}{l}P Q \text { interval } \\
\text { (1-ms increase) }\end{array}$ & 1.00 & $1.00-1.01$ & 0.43 & & & & 1.01 & $1.00-1.02$ & 0.031 & 1.00 & $1.00-1.01$ & 0.21 \\
\hline Adaptive CRT & 0.084 & $0.0045-0.46$ & 0.0023 & 0.12 & $0.006-0.69$ & 0.015 & 0.17 & $0.026-0.61$ & 0.0043 & 0.30 & $0.044-1.21$ & 0.095 \\
\hline
\end{tabular}

Ather abbreviations as in Tables 1,4.

optimization improves LV filling time. ${ }^{19}$ Therefore, periodic optimization of the AV delay is essential to enhance the effect of CRT, and the aCRT algorithm may be effective because of its periodic automatic evaluation function.

\section{aCRT and Mildly Wide QRS or Non-LBBB}

Although CRT is less effective for patients with non$\mathrm{LBBB}^{20}$ or mildly wide QRS morphology, ${ }^{21,22}$ the aCRT algorithm reduced the risk of cardiac death and HF hospitalization in our data. Regarding patients with a mildly wide QRS, a previous report, which was a subanalysis of the aCRT trial, showed that the aCRT algorithm could significantly improve the clinical composite score of patients with a mildly wide QRS. ${ }^{23}$ In patients with a wide $\mathrm{QRS}$, the positive effect of $\mathrm{BiV}$ pacing from the restoration of LV mechanical synchrony overwhelms the potential negative effect of RV pacing because the QRS duration correlates positively with the degree of electrical dyssynchrony. The positive effect of CRT in patients with mildly wide QRS is limited, however, because the degree of electrical dyssynchrony is smaller and contributes less to the progression of LV dysfunction than in patients with a wide QRS. In patients with a mildly wide QRS, the degree of electrical dyssynchrony at baseline may be smaller, and because of RV pacing, the potential detrimental effect of $\mathrm{BiV}$ pacing might be equal to or even greater than the positive effect of $\mathrm{BiV}$ from the restoration of $\mathrm{LV}$ mechanical synchrony. Accordingly, the aCRT algorithm, which reduces unnecessary RV pacing, preserves the positive net effect of CRT and results in better clinical outcomes for patients with a mildly wide QRS. In a previous report regarding the aCRT algorithm in patients with a mildly wide QRS, however, there were no significant differences in the combined endpoint of HF hospitalization or allcause death between the aCRT arm and the control arm (echo-optimized BiV CRT). ${ }^{23}$ One of the differences in comparison with our study was the proportion of patients with LBBB. The previous study ${ }^{23}$ included only LBBB patients who are supposed to respond to CRT, so there was no significant difference in the combined endpoint between the 2 groups. In patients with LBBB, the beneficial effect of CRT may be promised, even with comprehensive echocardiographic optimization, if we can deliver a LV lead to an appropriate site.

In this study, there were significant differences in the prevalence of IVCD and the degree of QRS width among patients with non-LBBB morphology (Table 3). A high prevalence of complete right bundle branch block (CRBBB) in the non-adaptive group might relate to the inferior effect of CRT compared with the adaptive group, because previous studies have shown that the response to CRT in patients with CRBBB is inferior to those with IVCD..$^{24,25}$ However, our data indicated no significant differences in cardiac death or HF hospitalization between the patients with RBBB and those with IVCD, both in the adaptive and the non-adaptive group. As for QRS width, generally, CRT is beneficial for patients with a wide QRS because it indicates the presence of electrical dyssynchrony. Among the patients with limited non-LBBB, the non-adaptive group had a wider QRS duration; therefore CRT was thought to be more beneficial for them. However, the clinical benefits of CRT were significantly higher in the adaptive group compared with the non-adaptive group. We supposed that the aCRT algorithm, which provides periodic optimization of $\mathrm{AV}$ and $\mathrm{VV}$ delay, was able to preserve appropriate pacing timing continuously in addition to reducing $\mathrm{RV}$ pacing, which impairs the physiological activation pattern, generating more electrical dyssynchrony.

\section{Clinical Implications}

Although CRT is essential therapy for HF patients with prolonged QRS duration, some patients do not respond to it, especially those with a mildly wide QRS or non-LBBB morphology. There were no severe complications related 
to use of the aCRT algorithm, such as significant battery consumption or induction of VAs. The aCRT algorithm may be an important tool in the management of patients with a mildly wide QRS or non-LBBB morphology.

\section{Study Limitations}

This was a single-center retrospective study, so the relatively small number of patients represents the main limitation. Compared with previous studies, the prevalence of ICM patients was relatively small, and as for baseline characteristics of patients with non-LBBB, there were significant differences in the prevalence of IVCD and degree of QRS width between the adaptive and non-adaptive groups. Nevertheless, no previous studies have evaluated the efficacy of the aCRT algorithm for patients with a mildly wide QRS or non-LBBB. Further, prospective multicenter studies including a larger number of patients with CRT devices using the aCRT algorithm are warranted to confirm these findings.

\section{Conclusions}

The new aCRT algorithm significantly reduced the risk of death and/or HF hospitalization, even in patients with a mildly wide QRS.

\section{Funding}

This study was supported by the Intramural Research Fund (25-4-7, K. Kusano) for Cardiovascular Diseases from the National Cerebral and Cardiovascular Center.

\section{Disclosures}

K. Kusano and T. Noda received honoraria for lectures from Medtronic Japan Co., Ltd.

\section{References}

1. Cleland J, Freemantle N, Ghio S, Fruhwald F, Shankar A, Marijanowski M, et al. Predicting the long-term effects of cardiac resynchronization therapy on mortality from baseline variables and the early response: A report from the CARE-HF (Cardiac Resynchronization in Heart Failure) Trial. $\mathrm{J} \mathrm{Am} \mathrm{Coll} \mathrm{Cardiol}$ 2008; 52: 438-445.

2. Goldenberg I, Moss AJ, Hall WJ, Foster E, Goldberger JJ, Santucci P, et al. Predictors of response to cardiac resynchronization therapy in the Multicenter Automatic Defibrillator Implantation Trial with Cardiac Resynchronization Therapy (MADIT-CRT). Circulation 2011; 124: 1527-1536.

3. Daubert C, Behar N, Martins RP, Mabo P, Leclercq C. Avoiding non-responders to cardiac resynchronization therapy: A practical guide. Eur Heart J 2017; 38: 1463-1472.

4. Mullens W, Grimm RA, Verga T, Dresing T, Starling RC, Wilkoff BL, et al. Insights from a cardiac resynchronization optimization clinic as part of a heart failure disease management program. J Am Coll Cardiol 2009; 53: 765-773.

5. Krum H, Lemke B, Birnie D, Lee KL, Aonuma K, Starling RC, et al. A novel algorithm for individualized cardiac resynchronization therapy: Rationale and design of the Adaptive Cardiac Resynchronization Therapy trial. Am Heart J 2012; 163: $747-752$.

6. Starling RC, Krum H, Bril S, Tsintzos SI, Rogers T, Hudnall JH, et al. Impact of a novel adaptive optimization algorithm on 30-day readmissions: Evidence from the Adaptive CRT Trial. JACC Heart Fail 2015; 3: 565-572.

7. Khaykin Y, Exner D, Birnie D, Sapp J, Aggarwal S, Sambelashvili A. Adjusting the timing of left-ventricular pacing using electrocardiogram and device electrograms. Europace 2011; 13: $1464-1470$.

8. van Gelder BM, Bracke FA, Meijer A, Pijls NH. The hemodynamic effect of intrinsic conduction during left ventricular pacing as compared to biventricular pacing. J Am Coll Cardiol 2005; 46: $2305-2310$.
9. Jones RC, Svinarich T, Rubin A, Levin V, Phang R, Murillo J, et al. Optimal atrioventricular delay in CRT patients can be approximated using surface electrocardiography and device electrograms. J Cardiovasc Electrophysiol 2010; 21: 1226-1232.

10. Ritter P, Padeletti L, Gillio-Meina L, Gaggini G. Determination of the optimal atrioventricular delay in DDD pacing: Comparison between echo and peak endocardial acceleration measurements. Europace 1999; 1: 126-130.

11. Gorcsan J 3rd, Abraham T, Agler DA, Bax JJ, Derumeaux G, Grimm RA, et al. Echocardiography for cardiac resynchronization therapy: Recommendations for performance and reporting - A report from the American Society of Echocardiography Dyssynchrony Writing Group endorsed by the Heart Rhythm Society. J Am Soc Echocardiogr 2008; 21: 191-213.

12. Surawicz B, Childers R, Deal BJ, Gettes LS, Bailey JJ, Gorgels $\mathrm{A}$, et al. AHA/ACCF/HRS recommendations for the standardization and interpretation of the electrocardiogram. Part III: Intraventricular conduction disturbances - A scientific statement from the American Heart Association Electrocardiography and Arrhythmias Committee, Council on Clinical Cardiology; the American College of Cardiology Foundation; and the Heart Rhythm Society: Endorsed by the International Society for Computerized Electrocardiology. Circulation 2009; 119: e235-e240.

13. Stellbrink C, Breithardt OA, Franke A, Sack S, Bakker P, Auricchio A, et al. Impact of cardiac resynchronization therapy using hemodynamically optimized pacing on left ventricular remodeling in patients with congestive heart failure and ventricular conduction disturbances. J Am Coll Cardiol 2001; 38: 1957-1965.

14. Takaya Y, Noda T, Nakajima I, Yamada Y, Miyamoto K, Okamura H, et al. Electrocardiographic predictors of response to cardiac resynchronization therapy in patients with intraventricular conduction delay. Circ J 2014; 78: 71-77.

15. Martin DO, Lemke B, Birnie D, Krum H, Lee KL, Aonuma K, et al. Investigation of a novel algorithm for synchronized leftventricular pacing and ambulatory optimization of cardiac resynchronization therapy: Results of the Adaptive CRT Trial. Heart Rhythm 2012; 9: 1807-1814.

16. Wilkoff BL, Cook JR, Epstein AE, Greene HL, Hallstrom AP, $\mathrm{Hsia} \mathrm{H}$, et al. Dual-chamber pacing or ventricular backup pacing in patients with an implantable defibrillator: The Dual Chamber and VVI Implantable Defibrillator (DAVID) Trial. JAMA 2002; 288: $3115-3123$.

17. Kass DA, Chen CH, Curry C, Talbot M, Berger R, Fetics B, et al. Improved left ventricular mechanics from acute VDD pacing in patients with dilated cardiomyopathy and ventricular conduction delay. Circulation 1999; 99: 1567-1573.

18. O'Donnell D, Nadurata V, Hamer A, Kertes P, Mohamed U. Long-term variations in optimal programming of cardiac resynchronization therapy devices. Pacing Clin Electrophysiol 2005; 28(Suppl 1): S24-S26.

19. Shanmugam N, Prada-Delgado O, Campos AG, Grimster A, Valencia O, Baltabaeva A, et al. Rate-adaptive AV delay and exercise performance following cardiac resynchronization therapy. Heart Rhythm 2012; 9: 1815-1821.

20. Sipahi I, Chou JC, Hyden M, Rowland DY, Simon DI, Fang JC. Effect of QRS morphology on clinical event reduction with cardiac resynchronization therapy: Meta-analysis of randomized controlled trials. Am Heart J 2012; 163: 260-267.

21. Sipahi I, Carrigan TP, Rowland DY, Stambler BS, Fang JC. Impact of QRS duration on clinical event reduction with cardiac resynchronization therapy: Meta-analysis of randomized controlled trials. Arch Intern Med 2011; 171: 1454-1462.

22. Stavrakis S, Lazzara R, Thadani U. The benefit of cardiac resynchronization therapy and QRS duration: A meta-analysis. $J$ Cardiovasc Electrophysiol 2012; 23: 163-168.

23. Yamasaki H, Lustgarten D, Cerkvenik J, Birnie D, Gasparini M, Lee KL, et al. Adaptive CRT in patients with normal AV conduction and left bundle branch block: Does QRS duration matter? Int J Cardiol 2017; 240: 297-301.

24. Gervais R, Leclercq C, Shankar A, Jacobs S, Eiskjaer H, Johannessen A, et al. Surface electrocardiogram to predict outcome in candidates for cardiac resynchronization therapy: A sub-analysis of the CARE-HF trial. Eur J Heart Fail 2009; 11: 699-705.

25. Bilchick KC, Kamath S, DiMarco JP, Stukenborg GJ. Bundlebranch block morphology and other predictors of outcome after cardiac resynchronization therapy in Medicare patients. Circulation 2010; 122: 2022-2030. 